FORMATION Formation emploi

Revue française de sciences sociales

136 | octobre-décembre 2016

Pêle-mêle

\title{
De l'activation à la précarité des demandeurs d'emploi : deux dispositifs en Belgique francophone
}

The activation to the insecurity of job seekers : two devices in French speaking part of Belgium.

Von der Aktivierung zur Prekarisierung von Arbeitssuchenden : zwei

Beschäftigungseinrichtungen im französischsprachigen Belgien

De la activación a la precariedad de los demandantes de empleo : dos dispositivos

en Bélgica francófona

Julie Gérard et Didier Vrancken

\section{CpenEdition}

Journals

Édition électronique

URL : http://journals.openedition.org/formationemploi/4930

DOI : 10.4000/formationemploi.4930

ISSN : 2107-0946

Éditeur

La Documentation française

Édition imprimée

Date de publication : 31 décembre 2016

Pagination : $99-119$

ISSN : 0759-6340

Référence électronique

Julie Gérard et Didier Vrancken, « De l'activation à la précarité des demandeurs d'emploi : deux dispositifs en Belgique francophone », Formation emploi [En ligne], 136 | octobre-décembre 2016, mis en ligne le 31 décembre 2018, consulté le 30 octobre 2020. URL : http://journals.openedition.org/ formationemploi/4930 ; DOI : https://doi.org/10.4000/formationemploi.4930 


\title{
De l'activation à la précarité des demandeurs d'emploi : deux dispositifs en Belgique francophone
}

\author{
JULIE GÉRARD \\ Doctorante en sciences politiques et sociales à l'université de Liège (Belgique), Centre de \\ recherche et d'interventions sociologiques (CRIS) \\ Didier VRANCKEN
}

Professeur de sociologie à l'université de Liège (Belgique), Centre de recherche et d'interventions sociologiques (CRIS)

Résumé

De l'activation à la précarité des demandeurs d'emploi : deux dispositifs en Belgique francophone

Cet article analyse deux dispositifs d'activation en Belgique francophone (une entreprise de formation par le travail et une agence de titres-services). Il illustre combien le croisement de politiques d'emploi à vocation universelle avec des politiques particularistes destinées aux personnes les plus fragiles fait émerger de véritables trappes à précarité. Cela soulève la question du développement progressif d'un nouveau régime de protection, opérant $a$ minima auprès des publics peu qualifiés, face à la menace que font peser le marché noir et plus largement l'informalité du travail.

Mots clés : chômeur, formation des chômeurs, mesure pour l'emploi, politique sociale, réinsertion professionnelle, accompagnement professionnel, Belgique

Abstract

The activation to the insecurity of job seekers: two devices in French speaking part of Belgium.

The article analyses two activation devices in the French-speaking part of Belgium (a company of training through work and a service vouchers firm). It illustrates how, crossing employment policies of universal scope with particularistic policies for the most vulnerable people, brings out true traps of insecurity. This raises the question of the progressive development of a new regime of protection, operating at least, with the low qualified people facing the threat posed to the black market and, more broadly, labor informality.

Keywords: unemployed person, training for the unemployed, employment scheme, social policy, re-integration into the labour market, coaching, Belgium

Journal of Economic Literature: J 68, J 24

Traduction : Auteur-e-s. 
En Belgique, les politiques actives de l'emploi ont émergé vers la fin des années 1990. Elles se définissent comme un ensemble de politiques publiques encourageant le développement de l'employabilité ou la capacité d'insertion socioprofessionnelle des demandeurs d'emploi afin qu'ils se rapprochent du marché du travail (Orianne, 2006). Elles s'inscrivent dans le cadre de la stratégie européenne ${ }^{1}$ et visent à atteindre un taux d'emploi de 73,2 \% d'ici 2020. Ces politiques reposent sur plusieurs fondements. D’une part, elles s'appuient sur l'expérience des pays nordiques qui ont fait de l'activation de l'emploi un élément clé de la compétitivité économique et de la lutte contre le chômage (Kvist, 2003). D’autre part, elles sont influencées par les théories économiques du chômage des années 1980 qui visent à faire "baisser le cô̂t du travail et accrôttre la concurrence sur le marché du travail, par l'affaiblissement de la protection de l'emploi des travailleurs intégrés et une meilleure employabilité des chômeurs "² (Erhel, 2012, p. 31).

Fers de lance de la politique de l'État social actif, dès leur origine, ces politiques d'activation ont visé la lutte contre le chômage de longue durée et l'exclusion sociale par un retour à l'emploi. Cherchant à colmater les brèches du système assurantiel via une activation de l'aide sociale et de l'allocation chômage (Hamzaoui, 2007), elles ont offert une innovation singulière sur laquelle nous souhaiterions insister. En opposition au welfare regim (Desmarez, 2003), elles ont ainsi proposé une articulation de politiques à vocation universelle (les politiques d'assurance sociale) à des politiques particularistes (les politiques d'aide sociale héritières des politiques d'assistance sociale) destinées aux personnes les plus fragiles.

Plus globalement, pour devenir actives, les politiques d'emploi ont puisé aux racines mêmes de l'action et de l'intervention sociales. Il s'est ainsi agi de prendre appui sur toute une constellation de services, d'intervenants et de réponses particularistes destinés à l'origine aux publics les plus vulnérables, là où l'individuation des interventions avait été expérimentée à la marge, au niveau du social assistanciel. Rappelons que ce social s'est déployé dans le sillon de la sphère de l'assistance, héritière de la prise en charge de l'indigence (loi du 10 mars 1925 organisant les Commissions d'assistance publique). Reposant sur des enquêtes sociales préalables menées auprès des personnes en difficulté, ce social particulariste et non contributif s'appuie sur des transferts sélectifs effectués sous conditions (âge, nationalité, résidence, ressources, disposition au travail). S'adressant à l'origine à des personnes précarisées dédouanées de l'obligation de travailler, il s'est largement étendu à la mise à l'emploi (Vrancken, 2010). Ainsi, alors que les politiques liées à l'État social avaient veillé à opérer une césure nette, un " grand partage ", au sein de la société, entre les salariés relevant des politiques d'assurance sociale et les personnes en situation de précarité sociale, les nouvelles mesures ont privilégié des politiques plus subjectives et plus interventionnistes alliant les deux régimes.

1. La Stratégie de Lisbonne (2000), la Stratégie Europe 2020 (EU2020).

2. Elle se réfère à la " capacité individuelle à garder un emploi, à en changer et à construire sa propre carrière ", (Lesfresnes, 1999, p. 467). 
Ces politiques d'activation ont eu pour caractéristique de consacrer l'argent public, et en particulier celui de l'assurance chômage ou de l'assistance sociale, au financement de l'emploi plutôt qu'au versement passif d'allocations de remplacement (Barbier, 2002). Outre cette conversion des allocations, l'activation a aussi porté sur les comportements de recherche d'emploi des individus par des dispositifs d'incitation et des sanctions financières (Béraud et Eydoux, 2009). Par cette double activation, les politiques actives d'aujourd'hui reposent davantage sur une conception interactive de l'employabilité puisqu'elles reflètent la " capacité relative d'un individu à obtenir un emploi compte tenu de l'interaction entre ses caractéristiques individuelles et le marché du travail" (Gazier, 2006, p. 421).

En Belgique, divers dispositifs d'activation ont ainsi vu le jour, afin de satisfaire à ces nouvelles orientations. À titre d'illustration, nous proposons l'analyse de deux cas (voir encadré 1) : une entreprise de formation par le travail ${ }^{3}$ (Synergie) et une agence de titresservices $^{4}$ (Proximity) $^{5}$. Alors que le premier dispositif tend à répondre à l'augmentation constante du taux de chômage des jeunes infra-qualifiés ${ }^{6}$, en misant sur une insertion socioprofessionnelle ${ }^{7}$, le second dispositif vise les besoins sociaux liés à la participation massive des femmes au marché du travail. Par leurs actions, tous deux répondent aux objectifs des politiques d'activation. En effet, ils permettent, d'une part, de rencontrer les besoins non satisfaits par les circuits de travail réguliers et, d'autre part, d'offrir aux chômeurs de longue durée la possibilité de reprendre contact avec le marché du travail, dans une logique de responsabilisation individuelle.

Pour ce faire, Synergie inscrit son action dans le workfare anglo-saxon (Bonvin et Orton, 2009) - un "programme de mise au travail obligatoire, en contrepartie d'allocations" - en exigeant qu'un "travail» soit fourni en "contrepartie " de prestations sociales, comme évoqué par Handler (2003, cité par Barbier, 2006, p. 27). En effet, nous verrons que cette entreprise propose une formation en situation de travail réelle à des demandeurs d'emploi (DE)

3. Ce type d'entreprises propose une formation technique en situation réelle de travail, sur chantier ou en atelier, à des stagiaires en difficultés d'insertion, par un accompagnement individualisé. Contrairement aux associations intermédiaires, en France, les entreprises de formation par le travail ne mettent pas à disposition leurs candidats puisque leur mission est essentiellement formative. Aucun contrat de travail assorti d'une rémunération n’y est ainsi signé.

4. C'est un système subventionné (réduction d'impôt égale à $30 \%$ ) par les régions, sous la forme de coupons titres-service. Il permet à tout particulier domicilié en Belgique de payer, à un tarif avantageux (neuf euros par titre), des prestations d'aide ménagère (nettoyage, préparation de repas, petits travaux de couture, courses ménagères, repassage, transport de personnes à mobilité réduite). Ce système se rapproche largement du chèque emploi-service français, hormis au niveau des services à la personne qui y sont proposés et du montant de l'avantage fiscal.

5. Les noms des dispositifs ont été modifiés afin d'en garantir l'anonymat.

6. C'est-à-dire ne disposant ni du diplôme du 2ème cycle de l'enseignement secondaire ni du certificat d'enseignement secondaire inférieur ni d'un titre équivalent ou supérieur.

7. Via, notamment, une lutte simultanée contre la privation d'emploi (chômage) et la possible relégation sociale (exclusion). 
engagés sous le statut de stagiaire, mais toujours dépendants de politiques publiques assurantielles.

Proximity, pour sa part, répond davantage à la stratégie du " making work pay ", littéralement " rendre le travail attractif ", de l'OCDE (Organisation de coopération et de développement économiques) (Gazier et Zajdela, 2006). En effet, elle maintient le versement des allocations de chômage 8 tout en augmentant, notamment, le « salaire mensuel » des DE via des incitations financières individualisées (défraiements kilométriques, remboursement des frais de communications téléphoniques incitant à l'emploi, etc.). Autant de mesures qui valorisent le travail et évitent les risques inhérents au travail au noir, comme la précarité, la marginalisation et l'absence de protection sociale (Conter, 2005).

Nous tenterons de montrer qu'à travers cette dédifférenciation, les politiques d'activation de l'emploi débouchent sur l'émergence d'un nouveau régime de protection sociale. Un régime opérant a minima auprès des publics largement fragilisés à qui il s'agit désormais d'offrir des perspectives de parcours, de remobilisation de soi, plutôt qu'un basculement vers l'assistanat, voire dans cette zone tant décriée de l'informalité du travail, caractéristique d'un état de dérégulation où les politiques publiques ne parviennent que partiellement à encadrer l'ensemble des activités de travail. Proposer du parcours, en somme, plutôt que des places ou un "placement » sur le marché de l'emploi.

Aussi nous est-il apparu important de saisir les reconfigurations actuelles des politiques d'activation de l'emploi au sein de deux dispositifs : une entreprise de formation par le travail (EFT) et une agence de titres-services (T-S). Ces derniers, en dispensant une aide individualisée à un public défavorisé et en quête de sens dans le champ de l'emploi, articulent des politiques à vocation universelle à des politiques particularistes. Compte tenu des choix empiriques effectués autour de ces deux dispositifs, les constats dressés dans cet article ne peuvent être généralisés à l'ensemble des structures de l'accompagnement vers l'emploi. Néanmoins, ils permettent de saisir les enjeux actuels qui traversent le secteur de l'insertion socioprofessionnelle en Belgique francophone.

Larticle sera structuré en trois parties. La première décrira le dispositif des EFT en général, puis étudiera le cas de Synergie - une EFT liégeoise - pour illustrer enfin ses stratégies locales à l'égard de ses deux finalités (l'accompagnement psychosocial et l'activité marchande). La deuxième partie sera consacrée au dispositif des titres-services. Elle se structure de la même manière que la précédente (présentation générale du dispositif, examen empirique de Proximity et analyse de ses effets). Enfin, la dernière partie questionnera le travail réel opéré par les travailleurs de ces deux dispositifs sur les parcours des publics cibles. Ce questionnement nous permettra de dégager des pistes de discussion sur l'émergence progressive d'un nouveau régime de protection auprès des publics en situation de précarité.

8. Sous certaines conditions spécifiques, comme le type et la durée du contrat de travail/de formation. 


\section{Encadré 1 : Description du travail de terrain}

Le travail de terrain a été réalisé, entre février 2014 et juin 2014, auprès de deux dispositifs d'activation, en Belgique francophone (Région wallonne) : I'entreprise de formation par le travail, Synergie, et l'agence de titres-services, Proximity.

Cette étude s'appuie sur une recherche empirique qualitative reposant sur une collecte documentaire (cadre législatif, sites internet, conventions cadres, etc.), l'observation de situations d'interactions (70 individuelles et 10 collectives) et la passation de 37 entretiens (dont 29 au sein de Proximity et 8 chez Synergie) (*), d'une durée moyenne de 51 minutes. Alors que l'observation portait sur la mise en œuvre des politiques d'activation au sein des deux dispositifs, les entretiens semi-directifs ont été réalisés en fin de terrain avec l'ensemble des travailleurs (conseillers, assistants sociaux, responsables, consultants, etc.) des deux structures, afin d'approfondir et de confronter les informations recueillies par l'observation. Ces données ont été transcrites intégralement.

Une analyse de contenu, de type thématique, a ensuite été mobilisée pour saisir le rôle des travailleurs dans leur mission d'accompagnement et de suivi. Les thèmes tels que le contexte institutionnel, les pratiques d'accompagnement, les ressources/contraintes locales, les concepts de parcours et transition, etc., ont été abordés.

Le croisement de ces trois méthodes s'est opéré dans une "logique de complémentarité » (Peretz, 2004) afin de constituer des regards distincts et juxtaposés sur la mise en œuvre des politiques d'activation.

Par interactions individuelles, nous entendons les entretiens réalisés en face-à-face entre bénéficiaires et travailleurs de l'accompagnement (les entretiens de recrutement, les entretiens de suivi ou de coaching individuel ou encore les remises de titres-services). Les interactions collectives, quant à elles, correspondent soit aux séances de groupe où les bénéficiaires sont invités à interagir et à collaborer (formations à la recherche d'emploi, mises en situation, formation collective, etc.), soit aux réunions d'équipe organisées en interne, au sein de chaque organisme, afin de faire le point sur l'activité de la structure et la situation économique de celle-ci (conseil d'entreprise, réunions d'équipe, conseil de prévention et protection au travail, etc.)

$\left(^{*}\right)$ : S'il est vrai que des différences existent en termes de nombre de personnes interrogées au sein des deux dispositifs, nous souhaitons souligner l'exhaustivité des données. En effet, au sein des deux dispositifs, nous avons eu la possibilité d'interagir avec l'ensemble du personnel. Toutefois, nous regrettons de ne pas avoir pu accéder formellement au discours des bénéficiaires du programme de Synergie. Ce biais a néanmoins été comblé partiellement par des échanges informels, en fin d'observations ou lors des diverses pauses. 


\section{Les entreprises de formation par le travail pour lutter contre le chômage des jeunes infra-qualifiés}

Bien que l'émergence des entreprises de formation par le travail s'inscrive dans un contexte institutionnel et légal plus vaste, la mise en ouvre concrète des politiques actives de l'emploi, quant à elle, est largement dépendante de contingences locales et des publics ciblés par les structures.

\subsection{Contexte d'émergence des entreprises de formation par le travail en Wallonie : vers la redéfinition progressive des activités d'insertion}

Diverses initiatives locales ont été mises en œuvre afin de faire face à la hausse du chômage et à la marginalisation sociale des groupes précarisés. Caractérisées par une individualisation et une intervention croissantes auprès des publics cibles, elles ne visaient pas uniquement le retour à l'emploi, mais plus largement la lutte contre la pauvreté et l'exclusion sociale.

En se développant dès les années 1980, ces initiatives ont surtout concerné des personnes précarisées ou peu qualifiées (Henry et al., 2009). Dès lors, en permettant à un public en rupture avec le système éducatif traditionnel d'acquérir une formation, ces initiatives se sont déployées en marge des politiques publiques traditionnelles (Mertens, 2010 ) et ont progressivement contourné la législation pour exister. Toutefois, c'est seulement en 1995 que les entreprises de formation par le travail (EFT) apparaîtront via la promulgation d'un arrêté du gouvernement wallon relatif aux EFT.

Constituées sous la forme d'associations sans but lucratif (ASBL) ${ }^{9}$ ou émanant des Centres publics d'action sociale (CPAS) ${ }^{10}$, les EFT proposent un accompagnement socioprofessionnel temporaire, durant une période limitée de douze à dix-huit mois, pour des demandeurs d'emplois infra-qualifiés inscrits au service public régional wallon de l'emploi (FOREm) ou pour des stagiaires en difficultés sociales. Les EFT ont pour particularité d'assurer la formation des demandeurs d'emploi - engagés sous le statut de stagiaires mais toujours dépendants de politiques publiques assurantielles - en situation réelle de travail, en leur offrant un accompagnement psychosocial individualisé. Il s'agit de les inscrire dans un processus de (ré)intégration professionnelle et sociale, par le biais d'une activité productive.

Pour ce faire, les EFT recourent à une pédagogie spécifique alternant apprentissage théorique et formation par la mise en situation réelle de travail au sein d'entreprises

9. Associations sans but lucratif (Loi du 27 juin 1921, M.B. le 1er juillet 1921, p. 5409).

10. Centre public d'action sociale (Loi du 8 juillet 1976, M.B. le 05 août 1976, p. 9876). 
ou chez les particuliers (Grégoire, 2003). Durant le temps de formation, les stagiaires sont dispensés de recherche d'emploi et ne sont donc plus convoqués par l'Office national de l'emploi (ONEm). En plus du maintien de leur allocation de chômage, ils percevront une indemnité individuelle d'un euro brut par heure de formation prestée et bénéficieront d'un défraiement kilométrique et du remboursement des frais de garderie lorsqu'ils seront en activités externes.

\subsection{De l'accompagnement professionnel à l'insertion socioprofessionnelle? : le cas de Synergie}

Nous allons à présent aborder le cœur du sujet à travers le cas de Synergie. Après la présentation du cadre organisationnel de l'EFT, nous décrirons le travail de réinsertion opéré par les travailleurs de Synergie. Celui-ci nous permettra, ensuite, de saisir les injonctions contradictoires pesant sur la structure privée qui la conduisent non seulement à opérer une catégorisation au sein de son public cible, mais aussi à développer ses activités commerciales.

\subsubsection{Un accompagnement au parcours de vie au sein de Synergie}

LEFT Synergie est un dispositif d'activation créé en 1997, sous le statut juridique d'ASBL, en Province de Liège. Financée par la Région wallonne et la ville de Liège, cette EFT dispense des formations pré-qualifiantes d'une durée d'un an dans les métiers du bâtiment, dans trois filières spécifiques : aide-soudeur, peintre et ouvrier polyvalent. Désirant offrir un accompagnement individualisé, l'EFT accueille entre quatre et six stagiaires par filière, selon un système d'entrée permanente. 
Tableau 1 : Relevé et contextualisation des personnes rencontrées

\begin{tabular}{|c|c|c|c|c|c|c|}
\hline & Extrait & Date & Fonction & Diplôme & Genre & Ancienneté \\
\hline \multirow{8}{*}{ 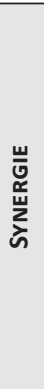 } & El 1 & mars-14 & Directeur & $\begin{array}{l}\text { Certificat d'enseignement secondaire inférieur } \\
\text { Formation « éducateur de rue » }\end{array}$ & H & 144 mois \\
\hline & El 2 & mars-14 & Assistant social & Bachelier assistant social & $\mathrm{F}$ & 144 mois \\
\hline & El 3 & févr-14 & Assistant comptable & Bachelier en comptabilité \& Master en fiscalité & $\mathrm{F}$ & 24 mois \\
\hline & El 4 & févr-14 & Formateur & Certificat de l'enseignement secondaire supérieur & H & 24 mois \\
\hline & El 5 & mars-14 & Formateur & Certificat de l'enseignement secondaire supérieur & H & 108 mois \\
\hline & El 6 & févr-14 & Formateur & Certificat de l'enseignement secondaire supérieur & $\mathrm{H}$ & 24 mois \\
\hline & El 7 & févr-14 & Educateur & Bachelier en art plastique & $\mathrm{F}$ & 36 mois \\
\hline & El 8 & févr-14 & Coordinateur & Bachelier en droit & $\mathrm{F}$ & 60 mois \\
\hline \multirow{29}{*}{ 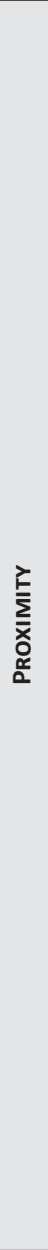 } & El 9 & mars-14 & Directeur & HEC & H & 84 mois \\
\hline & El 10 & mars-14 & Directeur & Master en psychologie & $\mathrm{F}$ & 84 mois \\
\hline & El 11 & mai-14 & Directeur & Bachelier en automation \& Master en management & $\mathrm{H}$ & 7 mois \\
\hline & El 12 & mai-14 & Directeur & Bachelier secrétariat & H & 18 mois \\
\hline & El 13 & mai-14 & Gestionnaire comptable & Bachelier comptabilité & $\mathrm{F}$ & 12 mois \\
\hline & El 14 & avr-14 & Responsable d'agence & Bachelier droit & $\mathrm{F}$ & 18 mois \\
\hline & El 15 & avr-14 & Responsable d'agence & Bachelier droit & $\mathrm{F}$ & 42 mois \\
\hline & El 16 & juin-14 & Consultant & Bachelier assistant social & $\mathrm{F}$ & 42 mois \\
\hline & El 17 & mai-14 & Consultant & Bachelier psychologie du travail & $\mathrm{F}$ & 5 mois \\
\hline & El 18 & mai-14 & Consultant & Bachelier éducateur spécialisé & $\mathrm{F}$ & 24 mois \\
\hline & El 19 & mai-14 & Responsable d'agence & Bachelier instituteur primaire & $\mathrm{F}$ & 54 mois \\
\hline & El 20 & mai-14 & Responsable d'agence & Bachelier en communication & $\mathrm{F}$ & 24 mois \\
\hline & El 21 & juin-14 & Responsable d'agence & Bachelier en relations publiques & $\mathrm{F}$ & 2 mois \\
\hline & El 22 & juin-14 & Responsable d'agence & Bachelier en relations publiques & $\mathrm{F}$ & 18 mois \\
\hline & El 23 & mai-14 & Aide-ménagère & Bachelier instituteur (trice) maternel(le) & $\mathrm{F}$ & 42 mois \\
\hline & El 24 & mai-14 & Aide-ménagère & Formation aide à domicile & $\mathrm{F}$ & 60 mois \\
\hline & El 25 & mai-14 & Aide-ménagère & Formation aide-soignante & $\mathrm{F}$ & 84 mois \\
\hline & El 26 & mai-14 & Aide-ménagère & Bachelier en puériculture & $\mathrm{F}$ & 24 mois \\
\hline & El 27 & mai-14 & Aide-ménagère & Formation en couture & $\mathrm{F}$ & 72 mois \\
\hline & El 28 & mai-14 & Aide-ménagère & Bachelier en sciences de l'éducation & $\mathrm{F}$ & 24 mois \\
\hline & El 29 & mai-14 & Aide-ménagère & Certificat de l'enseignement secondaire supérieur & $\mathrm{F}$ & 3 mois \\
\hline & El 30 & mai-14 & Aide-ménagère & Certificat de l'enseignement secondaire supérieur & $\mathrm{F}$ & 24 mois \\
\hline & El 31 & mai-14 & Aide-ménagère & Formation en couture & $\mathrm{F}$ & 24 mois \\
\hline & El 32 & mai-14 & Aide-ménagère & Bachelier en puériculture & $\mathrm{F}$ & 24 mois \\
\hline & El 33 & mai-14 & Aide-ménagère & Bachelier en secrétariat & $\mathrm{F}$ & 24 mois \\
\hline & El 34 & mai-14 & Aide-ménagère & Certificat de l'enseignement secondaire supérieur & $\mathrm{H}$ & 3 mois \\
\hline & El 35 & mai-14 & Aide-ménagère & Certificat de l'enseignement secondaire supérieur & $\mathrm{F}$ & 24 mois \\
\hline & El 36 & juin-14 & Aide-ménagère & Formation en comptabilité & $\mathrm{F}$ & 24 mois \\
\hline & El 37 & juin-14 & Aide-ménagère & Certificat de l'enseignement secondaire supérieur & $\mathrm{F}$ & 62 mois \\
\hline
\end{tabular}

Source : Entretiens semi-directifs réalisés entre février et juin 2014. 
Bien plus que de préparer ses stagiaires à un métier, Synergie vise à offrir un accompagnement au parcours de vie. En effet, la formation dispensée par l'EFT ne débouche ni sur de l'emploi, ni sur une certification, mais bien sur une (re)mise en mouvement des stagiaires, dans une perspective, toujours aléatoire, de se rapprocher du marché du travail :

"On est fort dans le parcours professionnel et même en termes d'évolution sociale. On leur apprend déjà ce qu'attend un employeur. [...] On est au stade de leur expliquer ce qu'est un patron et comment il fonctionne. On leur apprend qu'il faut être ponctuel, poli, etc. On leur donne toutes les armes sociales; la base, quoi. " (Extrait de l'observation d'une réunion, EI 2 ${ }^{11}, 7$ mars 2014)

Pour ce faire, Synergie offre aux stagiaires l'opportunité d'accéder à des formations préqualifiantes ou de valoriser l'expérience pressionnelle acquise durant leur accompagnement. L'EFT alterne une activité formatrice (cours théoriques et pratiques) et une activité commerciale (réalisation de chantiers de rénovation) permettant aux stagiaires de tester leurs compétences en situation réelle chez le particulier. Ainsi, Synergie vise davantage une situation de veille et de vigilance face aux aléas de la vie et aux risques inhérents aux parcours de leurs candidats que l'accès à un emploi. Par son accompagnement au parcours de vie, l'EFT invite ses stagiaires à se saisir d'eux-mêmes et à devenir responsables de leur employabilité.

\subsubsection{Un travail de (ré)insertion socioprofessionnelle en cinq temps}

Parallèlement à ces formations techniques et au travail productif, les travailleurs sociaux de l'EFT (éducateur, agent d'insertion, assistante sociale, etc.) opèrent un travail de (ré) insertion socioprofessionnelle par l'aide à l'élaboration d'un projet. Ce travail se compose de cinq phases et s'effectue à la demande du stagiaire.

Tout d'abord, la phase d'accueil vise à sensibiliser les demandeurs d'emploi intéressés par le dispositif d'activation, au cadre réglementaire qu'ils se préparent à suivre. Seuls les candidats répondant aux critères réglementaires ${ }^{12}$ et ayant défini un projet professionnel réaliste pourront accéder à l'accompagnement dispensé par Synergie. Ils seront invités à effectuer un bilan socio-professionnel avec l'assistant social et l'éducateur de l'EFT. Ce bilan évalue tant la situation sociale des candidats que leur niveau de compétences tech-

11. Par souci d'anonymisation, les extraits d'entretien seront désignés par les lettres EI (extrait d'interview) et un chiffre correspondant à l'ordre de passage des personnes rencontrées, qui sont présentées dans le tableau 1.

12. À savoir : être inscrit comme demandeur d'emploi au FOREm ; être éloigné de l'emploi ; être peu ou pas qualifié et avoir au maximum le Certificat d'enseignement secondaire inférieur ou être sous dérogation. Par éloigné de l'emploi, le FOREm entend des personnes qui, par des facteurs tant personnels (trajectoires de vie, assuétudes, maladies chroniques et/ou mentales, etc.) que professionnels (qualification, connaissance, expérience, durée du chômage, etc.) sont durablement exclues du marché du travail et qui nécessitent un accompagnement spécialisé (https://www.leforem.be/MungoBlobs/402/317/20100712_PEE_PHASE-2_ FINAL.pdf, consulté le 23/02/2016). 
niques. Les autres seront renvoyés soit vers le FOREm, soit vers un organisme d'orientation professionnelle.

L'inscription officielle des candidats, au sein de l'EFT, se fera en phase 2, au terme de ce bilan, par le biais d'un contrat de formation et d'une déclaration sur l'honneur attestant de leur engagement dans le dispositif. On perçoit ici combien les deux premières étapes offrent un véritable dispositif de filtrage et de sélection des publics, que cette sélection soit opérée par les professionnels ou par les stagiaires eux-mêmes.

Les conditions administratives remplies, le personnel de l'EFT peut alors faire connaissance avec ses candidats. Cette phase d'apprivoisement mutuel repose sur une proximité progressivement installée entre le formateur et son candidat. Lors de cette phase, l'interaction entre le stagiaire et le travailleur social prend généralement l'allure d'un entretien clinique où les formateurs posent un diagnostic à partir de l'observation directe de symptômes et de troubles d'employabilité (Orianne et Maroy, 2008). Des thèmes tels que la précarité, l'échec, la maltraitance, etc., sont abordés et discutés.

En phase d'activation, les formateurs auront pour tâche d'ouvrir les stagiaires à des perspectives plus actives (un plan d'actions, un projet professionnel, un nouveau départ, etc.) afin de leur permettre de se rapprocher du marché du travail. Les stagiaires seront invités à s'impliquer progressivement dans la construction de leur parcours en se montrant réceptifs aux exercices - techniques (réalisation de chantiers, soudure, etc.), professionnels (réalisation d'un Curriculum Vitae et d'une lettre de motivation ; entrainement aux tests d'aptitude) ou personnels (aller chez le coiffeur, se raser, etc.) - proposés par l'EFT.

Enfin, la phase d'adéquation doit en principe permettre à Synergie de valider le travail d'accompagnement précédemment réalisé par le biais d'un stage en entreprise.

En fin de parcours, l'objectif du dispositif est non seulement de rendre les stagiaires capables d'agir de manière autonome et responsable, mais également de leur rendre confiance en leur démontrant qu'ils sont encore employables, en leur apprenant à se ressaisir et à se préoccuper d'eux, de leur vie et de leur parcours.

\subsubsection{De l'écrémage du public au développement des activités commerciales}

L'EFT a rapidement soutenu son objectif social d'insertion par le biais d'un système de subventionnement incitatif (Lemaître et al., 2005). Ainsi, afin d'assurer son activité, Synergie a investi dans le développement de ressources publiques, faisant des appels à projets une de ses principales ressources financières. Toutefois, ce mode de financement étant fortement dépendant des réglementations et des processus institutionnels, l'entreprise s'est longtemps limitée à un strict respect des procédures institutionnelles (reporting, gestion par les objectifs, conformité aux méthodes préconisées par les décrets et l'agrément, etc.) plutôt qu'à l'innovation et à l'investissement réel dans l'accompagnement psychosocial de ses stagiaires. 
Parallèlement, et face à la demande grandissante, les formateurs de l'EFT ont progressivement délaissé l'aspect pédagogique pour se concentrer davantage sur leur activité marchande : le suivi de chantiers de rénovation chez le particulier. Un formateur témoigne avec nostalgie du passé :

"Avant, si un [de nos stagiaires] ne voulait pas faire un exercice ou un chantier, on s'asseyait et on discutait avec lui de son avenir, de comment faire connaissance, et on prenait le temps de tisser des liens de confiance pour les porter. Mais maintenant, essaie un peu de faire ça en plein milieu du salon d'un client. Déjà, il nous dira "et je vous paie pour bosser pas pour parler". Ils savent qu'on est une EFT, mais ils ne savent pas comment on agit dans une EFT. Je peux encore le faire ici, de temps en temps, mais encore... Ça devient impossible avec les chantiers. " (EI 24)

Cette activité permet à la structure d'assurer une rentrée financière régulière en plus de l'octroi ponctuel de subventions publiques.

Destiné initialement aux « cas sociaux " (drogués, sans-abris, jeunes de la rue, marginaux, etc.) ou aux publics relevant plutôt de l'aide sociale (bénéficiaires d'un revenu d'intégration du CPAS), Synergie a très vite durci ses critères d'inscription en ne sélectionnant que les " moins éloignés du marché du travail» (EI 4). Par cette expression, les formateurs entendent les demandeurs d'emploi inscrits au FOREm et ayant fait leurs preuves. En effet, le manque de subventions et les contraintes du secteur (restrictions budgétaires, élargissement des frontières au secteur marchand, etc.) ont contraint l'ASBL à développer davantage ses activités marchandes et à ne recourir qu'aux stagiaires possédant un minimum de compétences techniques et professionnelles ${ }^{13}$.

D'un soutien aux jeunes en difficulté à un centre de formation pré-qualifiante, l'EFT est devenue, au fil du temps, une réelle entreprise de rénovation répondant aux demandes des particuliers et devant se positionner de manière concurrentielle au sein du secteur du bâtiment. Le prix à payer d'un tel repositionnement fut bel et bien le rejet des cas trop lourds ou réputés «irrécupérables» (EI 1).

Ainsi, de manière relativement attendue, on observe que les formateurs de l'EFT se trouvent en porte-à-faux entre deux finalités diamétralement opposées : l'accompagnement psychosocial des demandeurs d'emploi, d'une part, et la survie financière de Synergie, d'autre part. Visant désormais principalement le maintien, voire la survie de la structure, les formateurs techniques préfèrent cumuler les chantiers et varier les tâches techniques plutôt que se cantonner au respect du programme de cours, en atelier.

Coincés entre des impératifs techniques, financiers, d'une part, et des normes et des règles publiques fortement contraignantes, d'autre part, les travailleurs de l'EFT semblent opérer un véritable tri parmi les publics auxquels Synergie était initialement destinée (Levêque et Vrancken, 2004). Il s'agit de devenir une entreprise du bâtiment rentable, faisant passer

13. À savoir : la maitrise des termes techniques, le maniement des outils, la compréhension orale du français, etc. 
ses missions de réinsertion sociale au second plan. Mais les facteurs liés au marché, au fonctionnement et aux règles publiques sont cependant insuffisants pour éclairer la dynamique à l'œuvre au cœur de ce dispositif. En nous référant à l'article de Boanca-Deicu (2016), nous pourrions supposer que les écarts de pratiques des formateurs de l'insertion seraient liés au contexte socio-économique du champ de l'intervention. Dès lors, ceuxci marquent le travail des formateurs d'une obligation de résultats dans un contexte de menace généralisée sur l'emploi. La finalité de leur travail se réduirait ainsi à des tâches de gestion administrative et financière.

Le dispositif, malgré ses limites et ses effets pervers (logique d'écrémage) apparaît alors comme un rempart, un filtre pour "changer sa vie " (EI 8), retrouver "le bon chemin" (EI 5), accéder à " un nouveau paysage" (EI 1), au prix d'une mise à l'épreuve de soi qui vise, in fine, moins le retour à l'emploi que la mise en mouvement, le fait de renouer avec les règles, les contraintes réaffirmées, comprises et acceptées.

\section{Le dispositif des titres-services : entre un objectif de réinser- tion socioprofessionnelle et des exigences de rentabilité}

Autrefois octroyés par les agences locales pour l'emploi, les titres-services (T-S) ont connu une nette évolution, ces dernières années, pour devenir un réel catalyseur sur le marché de la réinsertion socioprofessionnelle. Malgré cette popularité, le cadre légal des titres-services ne cesse de se durcir, ce qui entraîne des modifications de la nature du travail opéré par les consultants.

\subsection{Emergence et contexte de création des titres-services : une démocratisation qui interroge}

C'est en 2004 que les T-S sont créés par le gouvernement fédéral belge, afin de remplacer progressivement les agences locales (ALE) pour l'emploi, appelées à disparaître. Bien que ce dispositif se déploie toujours dans le secteur des services de proximité et repose sur un système de coupon (papier ou électronique), la portée diffère largement. Initialement, les ALE étaient exclusivement destinées aux chômeurs, alors que l'action des T-S brasse un public plus large, constitué à la fois de demandeurs d'emploi et de salariés peu qualifiés en attente d'un véritable contrat de travail dans le domaine ménager. À l'origine, ce dispositif d'activation a été conçu pour répondre à un objectif de régularisation des situations de travail. Ainsi, visant l'octroi d'un emploi de qualité en offrant aux aides ménagères "des garanties en termes de volume de travail à effectuer, mais aussi en termes de régularité des revenus" (Conter, 2005, p. 267), ces agences 
de T-S, reconnues par un agrément fédéral ${ }^{14}$, répondaient aux attentes de la régulation publique en termes de résorption du travail au noir, d'activation des demandeurs d'emploi et d'insertion socioprofessionnelle, via l'octroi d'un contrat de travail décent.

Néanmoins, en réalité, le travailleur prestant sous T-S conclut certes un contrat de travail à durée indéterminée, mais de treize heures minimum, avec une agence de T-S agréée qui agit comme employeur juridique. En général, si la personne répond aux attentes de l'employeur en termes d'efficacité et de rentabilité, elle aura la possibilité de passer à temps partiel $(26 \mathrm{~h})$, puis à temps plein $(38 \mathrm{~h})$ par le biais d'avenants ${ }^{15}$. En attendant, le travailleur prestant sous T-S, à temps partiel, peut demander le maintien des droits à l'ONEm afin de bénéficier de l'allocation de garantie de revenus en plus de son salaire ${ }^{16}$ et des divers soutiens financiers alloués par l'agence.

Six types de services de proximité, répartis en deux catégories - à domicile (le nettoyage, la préparation de repas, la lessive et le repassage du linge) et hors domicile (les petits travaux de couture, le transport de personnes à mobilité réduite et les courses) sont officiellement autorisés par le gouvernement fédéral. Ainsi, l'État conserve son rôle de financeur et de régulateur de politiques publiques alors qu'il en délègue totalement la mise en œuvre à une pluralité d'opérateurs.

En effet, le champ de l'aide à domicile de nature ménagère s'est rapidement ouvert à une gamme de prestataires, qu'ils soient publics (CPAS, Communes), privés lucratifs (société privée à responsabilité limitée, société anonyme dont les sociétés d'intérim, les personnes physiques) ou d'économie sociale (sociétés à finalité sociale, ASBL, etc.) alors qu'à l'époque des ALE, seules les entreprises d'économie sociale pouvaient se constituer en agence de T-S.

\subsection{Vers un service de proximité : le cas de Proximity}

Après avoir rendu compte de la double mission de Proximity, nous illustrerons les difficultés rencontrées par les consultants de l'entreprise de T-S pour articuler un objectif d'efficacité et de rentabilité à une mission sociale de réinsertion. Enfin, nous verrons les effets produits par cette situation sur le parcours des aides ménagères.

14. Loi du 20 juillet 2001 visant à favoriser le développement de services et d'emplois de proximité (M.B. le 11 août 2011, p. 27453).

15. Chaque heure supplémentaire « au-delà des treize heures par semaine » est ajoutée par avenant.

16. Celui-ci est payé en partie par l'utilisateur (tout citoyen domicilié en Belgique) sous la forme de coupon titre-service d'une valeur de neuf euros (depuis le 1er janvier 2014) et par le gouvernement fédéral (intervention forfaitaire). 


\subsubsection{La double mission de Proximity: régulariser et/ou réinsérer?}

Proximity ${ }^{17}$ est une entreprise de T-S créée en 2007, en Région liégeoise (Belgique francophone), sous le statut juridique de société privée à responsabilité limitée, à finalité lucrative. Bien plus que de générer de l'emploi pour les personnes peu qualifiées, cette entreprise de T-S vise à répondre efficacement aux demandes des particuliers en termes de services de proximité et d'appui administratif. Afin d'assurer sa mission de réinsertion via l'aide à domicile de nature ménagère, l'entreprise a décidé de se focaliser sur quatre activités : le ménage, le repassage, le lavage de vitres et les courses.

Bien que financée partiellement par le gouvernement (réduction d'impôt de 30 \%), l'entreprise assure la viabilité financière de sa structure grâce à un recrutement sélectif et à un placement rapide. Pourtant, depuis le mois d'août 2012, les entreprises de T-S se voient contraintes, dans leurs recrutements, de respecter un certain quota d'embauche. En effet, $60 \%$ de leurs recrutements doivent dorénavant être réalisés parmi des chômeurs complets indemnisés ${ }^{18}(\mathrm{CCI})$ et/ou des bénéficiaires de l'aide sociale via le revenu d'intégration ${ }^{19}$, ce qui a totalement modifié le travail des consultants en T-S ainsi que l'objectif initial de la structure : l'entreprise n'œuvre plus tant à un objectif de régularisation des situations qu'à un objectif de réinsertion - en désaccord a priori avec ses missions de base :

"C'est de plus en plus commercial. [...] Même si on a toujours un objectif de réinsertion car on nous demande d'engager des personnes chômeuses de longue durée. On nous demande ces $60 \%$. Mais ça reste toujours social, mais pour la rentabilité et la vie de la société, le commercial prend le dessus. Et je le ressens beaucoup ... mais c'est mon opinion. " (EI 15)

\subsubsection{L'activité de placement de Proximity: un objectif d'efficacité et de rentabilité}

Les aides ménagères désirant travailler au sein de Proximity sont invitées à soumettre leur candidature par voie postale ou par courrier électronique. Celles qui auront satisfait à l'entretien téléphonique et, in fine, répondu aux critères de l'entreprise ${ }^{20}$, seront convoquées

17. En 2014, Proximity comptait 480 membres au sein de son personnel, dont une vingtaine faisait partie du staff administratif.

18. Selon l'ONEm, les chômeurs complets indemnisés regroupent les demandeurs d'emploi qui ne sont pas liés par un contrat de travail, c'est-à-dire "les chômeurs indemnisés demandeurs d'emploi après travail (à temps plein ou à temps partiel volontaire), et après études, les dispensés pour difficultés sociales et familiales ainsi que les chômeurs âgés et les prépensionnés à temps plein " (http://www.onem.be/fr/groupe-i-les-chomeurs-indemnises, consulté le 27/02/2016). En fonction de leur situation familiale et du motif de leur inscription au chômage, les CCI bénéficieront d'allocations de chômage différentes et dégressives dans le temps.

19. En Belgique, le revenu d'intégration est une aide sociale financière garantie accordée par les CPAS aux personnes n'ayant pas suffisamment de moyens de subsistance (Loi du 26 mai 2002, M.B. le 31 juillet 2002, p. 33610).

20. À savoir : disposer d'une aide à l'emploi, habiter dans un rayon de 20 kilomètres, avoir au moins une expérience probante dans les T-S, etc. 
pour un entretien de recrutement. Bien qu'aucun diplôme ne soit nécessaire, Proximity n'engage que des travailleuses ayant un minimum d'organisation et de condition physique. Lors de ce screening, de multiples questions relatives à leur expérience professionnelle et à leur motivation leur seront alors posées par les consultantes de l'entreprise : "Pourquoi voulez-vous cet emploi ? ", "A quelle heure êtes-vous prête à vous lever ? ", etc. (Extrait de l'observation d'entretiens de recrutement, EI18, 5 mai 2014). Néanmoins, ce n'est pas pour autant qu'elles seront automatiquement engagées par l'entreprise.

En effet, elles devront démontrer leur motivation lors d'un entretien de recrutement avec le responsable d'agence. Cet entretien a pour objectifs d'évaluer, d'une part, l'apparence physique de l'aide ménagère et de démontrer, d'autre part, le " sérieux " de Proximity en tant que prestataire de services de proximité. Ensuite, le responsable approfondira les informations recueillies par téléphone, afin d'apprécier les réelles motivations de l'aide ménagère. Enfin, si cette dernière est retenue, le recruteur posera différentes questions pratiques telles que les horaires souhaités, le type de contrat envisagé, etc. Si la personne correspond aux attentes de l'agence et au profil recherché, le responsable constituera son dossier administratif en veillant au respect de la procédure : réalisation du contrat de travail en deux exemplaires, déclaration DINOMA ${ }^{21}$, remise des documents officiels, etc.

Une fois la partie administrative réalisée, l'aide ménagère peut enfin être mise au travail. À l'issue de quelques semaines d'expérience, l'agence opèrera un suivi dit de "qualité "22 auprès de chaque client. Il consiste en un suivi téléphonique qui permet de résoudre directement les problèmes en cas d'avis défavorables.

Outre ce contrôle, un suivi de "rentabilité " - réalisé sur la base de statistiques établies à partir des heures prestées et des absences justifiées - a été instauré en 2013. Il sert non seulement d'appui à l'évaluation mensuelle des aides ménagères mais aussi à l'analyse de la gestion prévisionnelle des ressources humaines de l'agence. "En cas de résultat négatif, l'aide ménagère est avertie. Elle apparaît en orange dans le tableau et elle a trois mois pour faire ses preuves» (EI 15).

\subsubsection{D'un maintien dans la précarité à l'inactivité professionnelle}

Alors que Proximity assurait sa différence sur le marché des T-S par un recrutement sélectif et de qualité en n'engageant que des aides ménagères expérimentées - et souvent issues du circuit non déclaré -, l’entreprise a dû revoir ses critères de sélection afin de se conformer aux nouvelles exigences institutionnelles. En effet, face à l'obligation d'engager $60 \%$ de

\footnotetext{
21. La Dimona (Déclaration Immédiate) est un message électronique par lequel l'employeur communique toute entrée et sortie de service d'un travailleur à l'Office national de la sécurité sociale belge. Ce dispositif a été mis en place pour éviter le travail au noir dans le secteur de la restauration et de la construction (https:// www.socialsecurity.be/site_fr/employer/applics/dimona_new/index.htm, consulté le 27/02//2016).

22. Celui-ci se tient après la première prestation, puis ponctuellement grâce à un rappel automatique d'agenda ou en cas de problèmes récurrents.
} 
CCI ou de bénéficiaires du revenu d'intégration, les consultants de Proximity ont élargi leur champ d'action en se focalisant sur d'autres profils d'aides ménagères.

Initialement, la majorité des aides ménagères qui postulaient au sein de Proximity provenaient du travail au noir par choix. Motivées par un objectif de régularisation, ces dernières étaient à la recherche d'une insertion « durable » sur le marché de l'emploi et d'une sécurité d'emploi devant leur permettre de bénéficier d'une protection sociale. Considérées comme le " jackpot» (EI 21) aux yeux des consultants, ces aides ménagères apportaient leur portefeuille de clients qu'il ne restait plus qu’à légaliser. Que demander de plus pour l'entreprise que de "mettre en lumière et au clair le travail au noir, tant redouté par l'État" (EI 9) ? Mais à ce jour, ces aides ménagères ne représentent plus qu'un quart des effectifs.

Avec le changement de législation, on observe une prolifération de "mauvais profils " (EI 17) postulant par nécessité (les non-motivées, les cas sociaux, les exclus du chômage, etc.). Face à cette situation, les consultants ont dû revenir vers d'autres tâches plus psychologisantes qu'ils avaient pourtant volontairement écartées de leur mission : accompagnement de personnes, coachings individuels, conscientisation, etc. Outre une transformation du métier d'employés titres-services, cette contrainte chiffrée a eu, pour second corollaire, une pression aux résultats. L'atteinte des $60 \%$ était devenu l'objectif principal en termes de recrutement, quitte à évincer certains critères de sélection pourtant essentiels à la fonction (comme l'expérience dans le secteur) :

"Je le sens que c'est beaucoup plus "rentabilité" et j'ai du mal avec ça car je ne suis pas quelqu'un de rentable, commercial. Je comprends limportance de ça (les chiffres, etc.) mais voir par la rentabilité, je n'aime pas. Des fois, je le ressens fort et je le vis mal. " (EI 14)

Afin de satisfaire au quota trimestriel des $60 \%$ imposés par le gouvernement, certaines agences n'hésitent pas à engager, pour une courte durée, des aides ménagères répondant aux critères institutionnels, pour s'en défaire aussitôt. Une telle stratégie tend à maintenir les aides ménagères dans une "trappe à précarité " plutôt qu’à les réinsérer durablement sur le marché de l'emploi (Guillemot et al., 2002)23. En outre, cette recherche de chômeurs, destinée à répondre aux critères institutionnels et à éviter de recevoir un avertissement de l'ONEm, peut également avoir pour effet d'écarter certains CV intéressants. Tel est le prix à payer pour maintenir son agrément d'agence T-S.

Parallèlement aux mesures institutionnelles, depuis deux ans, la direction a imposé à chaque agence de ne recruter que des travailleuses bénéficiant en plus d'aides à l'emploi. Ces aides permettent à l'entreprise d'atteindre plus aisément son quota d'heures de placement à effectuer puisqu'elles prévoient une réduction des cotisations patronales de sécurité sociale en cas d'embauche d'un demandeur d'emploi. En outre, cette réduction est cou-

23. En effet, l'octroi temporaire de travail confine les aides ménagères dans un secteur secondaire composé de " mauvais emplois " souvent faiblement rémunérés, mais dont le montant de rémunération est toutefois supérieur à leur allocation de chômage. 
plée à une activation de l'allocation de chômage en cas d'embauche d'un chômeur complet indemnisé ou d'un bénéficiaire du revenu d'intégration sociale. Ce n'est donc plus le repérage d'aides ménagères les plus expérimentées qui s’opère, mais plutôt la recherche des chômeurs les plus rentables pour l'entreprise. Néanmoins, ce recours aux aides publiques décourage de plus en plus de demandeurs d'emploi à trouver un emploi, notamment en raison de la perte de " revenus d'assistance " résultant de l'obtention de l'emploi d'aide ménagère.

Il n'en demeure pas moins que la majorité des travailleuses qui se présentent - et qui répondent aux critères fédéraux - sont en difficulté sociale ou en instabilité conjugale :

"Je pense que l'on est plus, maintenant, dans de la réinsertion tout court que dans l'insertion du travail illégal dans du travail légal. On est plus dans un travail de réinsertion sociale. "(EI 18)

Réinsertion économique, réinsertion sociale, réinsertion " tout court " (EI 16) ? Pour des raisons décrétales et économiques, Proximity ne vise plus prioritairement la réinsertion sur le marché du travail, mais met dorénavant l'accent sur ce qui s'apparente plus à un travail sur les parcours, un travail de conscientisation et de réhabilitation de la personne.

\section{Un travail sur les parcours}

Créés dans la lignée du développement du discours sur l'État social actif, tant l'EFT que l'agence de T-S s'inscrivent clairement dans le cadre des politiques actives d'emploi en Belgique.

Au-delà de leur volonté de rendre confiance aux demandeurs d'emploi, via un accompagnement renforcé, les travailleurs de ces deux dispositifs d'activation tentent également de rapprocher les destinataires du marché du travail, en leur démontrant qu'ils sont encore employables. Toutefois, ce rapprochement semble s'opérer par un maintien dans un précariat social, voire dans une véritable trappe au chômage (Benarrosh, 2000). D'une part, Synergie offre l'opportunité aux stagiaires d'entrer dans un long cheminement vers l'emploi grâce à un accompagnement psychosocial et professionnel individualisé, mais insuffisant pour bénéficier d'un emploi et pour pouvoir s'inscrire sur le marché primaire (Doeringer et Piore, 1971). D’autre part, si dès le début de l'accompagnement, Proximity rend espoir aux demandeurs d'emploi en leur proposant un emploi protégé afin d'échapper au marché noir, force est de constater que cet emploi est souvent temporaire, faiblement rémunéré, et qu'il offre peu de perspectives professionnelles, mais des allers-retours incessants vers le chômage.

Ces deux dispositifs nous permettent d'illustrer l'existence de mécanismes de filtrage, de tri destinés à repérer et à retenir les profils jugés les plus « employables » ou les plus conformes aux critères institutionnels. En fonction des pressions réglementaires et juridiques éma- 
nant des pouvoirs publics et des contraintes budgétaires, une véritable sélection parmi les publics cibles s'opère bel et bien. Il s'agit de rendre effective la mission d'accompagnement des intervenants, tout en veillant à préserver les droits sociaux des destinataires. Par conséquent, il semblerait que cette lutte contre le chômage de longue durée et l'exclusion sociale passe non seulement par une mobilisation des demandeurs d'emploi et une activation des dépenses publiques, mais également par une flexibilité des conditions d'embauche et une dégradation des formes de stabilisation sur le marché de l'emploi (Supiot, 1999).

Ces constats rejoignent largement les résultats de l'étude internationale réalisée par Darmon et al. (2004) sur les organismes de formation d'aide aux plus vulnérables ou encore les travaux français de Benarrosh (2000 ; 2005), de Gratadour et Mansuy (2007) ainsi que de Ferracci (2007) sur l'évolution des services publics de l'emploi dans l'accompagnement des chômeurs. Tous concluent à une sélection des plus employables ou des moins éloignés du marché du travail et à une relégation des « inemployables " vers d'autres structures plus stigmatisantes.

À terme, une nouvelle zone combinant à la fois mesures universalistes et mesures plus " actives " et individualisées émergerait, offrant peu à un peu un nouveau régime de protections auprès de publics aidés, accompagnés et avant tout reconnus en situation de précarité (Eckert, 2010). Ce régime « faible " viserait tant à assurer des minima sociaux qu’à individualiser les interventions et à mobiliser les publics destinataires. Sans pouvoir garantir un retour à l'emploi, cette zone offrirait un maintien des droits sociaux, une protection sociale minimale, tout en incitant à la veille, au ressaisissement de soi, de son sort, de son niveau de formation. Elle proposerait d'entretenir une sensibilité permanente à l'état du marché et de la société pour éviter une relégation totale dans l'assistanat, voire dans le travail au noir et tout un monde de l'informalité (Lesemann, 2014) qui menaceraient désormais les pays occidentaux et pèseraient de plus en plus sur la régulation des systèmes protectionnels édifiés dans l'immédiat après-guerre.

\section{Conclusion}

Ainsi, les deux dispositifs analysés apparaissent-ils comme de véritables filtres ouvrant et fermant l'accès à un nouveau régime protectionnel organisé autour de l'entretien d'un "précariat social» (Castel, 2007), alliant des aides à l'emploi et des allocations diverses.

Par ce jeu d'accès ou de rejet, les deux dispositifs décrits permettent théoriquement tant un passage à l'emploi, un accès au dispositif, à une formation, une ouverture ou un maintien de droits au chômage, qu'une sortie vers le marché noir et le monde de l'informalité du travail.

Comme nous l'avons montré, si les stagiaires et les intervenants de Synergie évoquent bien l'existence de contraintes économiques et politiques qui pèsent sur la dynamique étudiée, les aides ménagères, les consultants, voire les responsables d'agence de Proximity illustrent également toute l'importance d'une zone d'ombre, voire d'un secteur mena- 
çant duquel il s'agirait de s'extraire pour trouver "une certaine sécurité" (EI 30), entrer "dans le système" (EI 33), "se faire une épargne pension" (EI 22). La menace planant sur le dispositif étant désormais "qu'en augmentant les titres, les personnes vont retourner vers le noir. Si on continue sur cette voie là, c'est ce qui va arriver" (EI 11). Ainsi, ces dispositifs destinés avant tout à activer les publics vers l'emploi offriraient la possibilité de mettre les personnes en mouvement, de produire du parcours, se déclinant à travers les transitions, les passages - accès ou sorties - vers l'emploi, la formation, le précariat ou le marché noir.

Dans ce contexte, toute réflexion sur un retour à l'emploi, sur la sortie du chômage, perdrait en pertinence. En effet, il s'agirait moins de franchir, sur un mode disjonctif, des limites, des frontières partageant le marché du travail et le chômage, le marché primaire et le marché secondaire, l'assurance chômage et l'emploi, l'assurance et l'assistance, que d'intégrer en permanence l'incertitude et l'aléa aux parcours de vie et d'emploi.

Bibliographie

Barbier J.-C. (2002), « Peut-on parler d' "activation" de la protection sociale en Europe ? ", Revue française de sociologie, vol. 43, 2, pp. 307-332.

Barbier J.-C. (2006) (avec des contributions de Sylla N.S. et Eydoux A.), Analyse comparative de l'activation de la protection sociale en France, Grande Bretagne, Allemagne et Danemark, dans le cadre des lignes directrices de la stratégie européenne pour l'emploi, Rapport pour la DARES (ministère du Travail), CEE, janvier.

Benarrosh Y. (2000), "Tri des chômeurs : le nécessaire consensus des acteurs de l'emploi », Travail et Emploi, 81, pp. 9-26.

Benarrosh Y., (2005), «Débat : Recherche d'emploi : quelle intermédiation ? Entre qui ? ... Et quoi ? ", Formation Emploi, 92, pp. 65-69.

Béraud M., Eydoux A. (2009), "Activation des chômeurs et modernisation du service public de l'emploi », Travail et Emploi, 119, pp. 9-21.

Boanca-Deicu I. (2016), "La formation à l'insertion, une activité de service à part ?", Formation Emploi, 134, pp. 67-87.

Bonvin J.-M., Orton M. (2009), "Activation policies and organisational innovation: the added value of the capability approach", International Journal of Sociology and Social Policy, vol. 29, 11/12, pp. 565-574.

Castel R. (2007), «Au-delà du salariat ou en deçà de l'emploi ? L'institutionnalisation du sous-emploi ? ", in Paugam S. (dir.), Repenser la solidarité : l'apport des sciences sociales, Paris, Presses Universitaires de France, pp. 416-433. 
Conter B. (2005), "Chapitre 8 : Agences locales pour l'emploi et titres-services. Entre développement de services et activation ", in Vielle P., Pochet P., Cassiers I. (2005), L'État social actif. Vers un changement de paradigme?, Bruxelles, Peter Lang.

Darmon I., Frade C., Demazière D., Haas I. (2004), "Formés et formateurs face à la "double contrainte" des programmes de formation à l'employabilité des chômeurs de longue durée ", Formation Emploi, 85, pp. 57-77.

Desmarez P. (2003), "La comparaison internationale des marchés du travail : pour une redéfinition des frontières ", L’Année sociologique, vol. 53, 2, pp . 345-371.

Divay S. (2009), « Nouveaux opérateurs privés du service public de l'emploi : les pratiques des conseillers sont-elles novatrices ? ", Travail et Emploi, 119, pp. 37-49.

Doeringer P., Piore M. (1971), Internal Labor Markets and Manpower Analysis, Lexington.

Eckert H. (2010), "Précarité dites-vous ? ", SociologieS [En ligne], Débats, La précarité, mis en ligne le 27 septembre 2010, consulté le 10 août 2015. URL : http://sociologies. revues.org/3285.

Erhel C. (2012), « Politiques de l'emploi : la tendance à l'activation donne-t-elle une place accrue à l'accompagnement ? ", Informations sociales, vol. 1, 169, pp. 30-38.

Ferracci M. (2007), "Améliorer le service public de l'emploi : ce que disent les faits ", Revue française d'économie, vol. 21, 3, pp. 75-135.

Gazier B. (2006), "L'employabilité », in Allouche J. (coord.), Encyclopédie des Ressources Humaines, Paris, Vuibert, $2^{\text {ème }}$ édition, pp. 350-356.

Gazier B., Zajdela H. (2006), "Making work pay” : from justications to implementations”, Cahiers de la Maison des Sciences Économiques, Paris, CNRS.

Grégoire O. (2003), "Profils nationaux des entreprises sociales d'insertion : Belgique", Working Paper Series, n 03/03, EMES European Research Network, Liège.

Guillemot D., Pétour P., Zajdela H. (2002), «Trappe à chômage ou trappe à pauvreté. Quel est le sort des allocataires du RMI ?", Revue économique, vol. 53, pp. 1235-1252.

Gratadour C., Mansuy M. (2007), « Sous-traiter l'accompagnement des chômeurs : éléments d'évaluation », Travail et Emploi, 112, pp.21-34.

Hamzaoui M. (2007), «Économie sociale d'insertion : enjeux et limites », Empan, vol. 1, 65, pp. 136-145.

Henry A., Nassaut S., Defourny J., Nyssens M. (2009), «Économie plurielle et régulation publique. Le quasi-marché des titres-services en Belgique "Société et Avenir, Politique scientifique fédérale, Academia Press. 
Kvist J. (2003), "Scandinavian Activation Strategies in the 1990s: Recasting Social Citizenship and the Scandinavian Welfare Model", Revue française des affaires sociales, vol. 4, 4, pp. 193-222.

Lefresne F. (1999), "Employability at the heart of the European Employment strategy", Transfer: European Review of Labour and research, vol. 5, pp. 460-480.

Lemaître A., Nyssens M., Platteau A. (2005), « Les entreprises sociales d'insertion par le travail entre idéal-type et institutionnalisation ", Recherches Sociologiques, vol. 01, pp. 129-152.

Lesemann F. (2014), «Agir face à l'incertain : le potentiel heuristique de l'informalité pour penser l'incertain. Quand le sud questionne le nord ", in Vrancken D. (dir.), Penser l'incertain, Québec, Presses de l'Université Laval, pp. 93-112.

Levêque A., Vrancken D. (2004), «La gestion par la dérogation. Une analyse de cas des politiques d'apprentissage industriel en Belgique francophone », Formation Emploi, 86, pp. 39-52.

Mertens S. (2010), La gestion des entreprises sociales, Edipro, Liège.

Orianne J.-F. (2006), « Politiques actives d'emploi et professionnels de l'employabilité : critique et clinique ", Travail Emploi Formation, vol. 6, pp. 53-92.

Orianne J.-F., Maroy C. (2008), «Esquisse d'une profession consultante : les intermédiaires du marché du travail en Wallonie », Formation Emploi, 102, pp. 21-39.

Peretz H. (2004), Les méthodes en sociologie. L'observation, Paris, La Découverte.

Supiot A. (1999), Au-delà de l'emploi, Paris, Flammarion.

Vrancken D. (2010), Social Barbare, Charleroi, Couleurs Livres. 


\section{RELATIONS INDUSTRIELLES}

Revue trimestrielle bilingue publiée depuis 1945 par le Département des relations industrielles de I'Université Laval

Tribute to Richard J. Long/ Hommage à Richard J. Long

ARTICLES

Quand le syndicat devient vecteur d'inégalités: les effets des clauses «orphelins» sur l'association syndicale DIANE GAGNÉ ET MARIE-JOSÉE DUPUIS

Safeguarding Workers: A Study of Health and Safety Representatives in the Queensland Coalmining Industry, 1990-2013

DAVID WALTERS, RICHARD JOHNSTONE, MICHAEL QUINLAN AND EMMA WADSWORTH

Pratiques de conciliation travail-famille et détresse psychologique des salariés québécois: une comparaison selon le genre MAUDE BOULET ET CÉLINE LE BOURDAIS

The Invisibilization and Denial of Work in Argentinian Garment Homework

ROSARIA BURCHIELLI AND ANNIE DELANEY

Analyse des composantes de la charge de travail perçue par les cadres dans un contexte de gestion des compétences NARGES SASSI ET HAZEM BEN AISSA

The Effects of the Psychological Contract among Professional Employees Working

in Non-Professional Organizations

PASCAL PAILLÉ, NICOLAS RAINERI ET PATRICK VALEAU

L'identification au contenu du travail comme déterminant du souhait de rester dans un emploi

\section{INDUSTRIAL RELATIONS QUARTERLY REVIEW}

A bilingual quarterly published since 1945 by the Industrial Relations Department, Université Laval

\section{RI/IR EN LIGNE}

RI/IR est disponible en ligne sur le site Erudit :

\section{www.erudit.org/revue/ri}

Pour un abonnement institutionnel, contacter Érudit.

Pour consulter les règles de publication ou pour vous abonner, visitez notre site Internet :

www.riir.ulaval.ca

\section{RI/IR ONLINE}

$R I / / R$ is available on line on

Erudit website at:

\section{www.erudit.org/revue/ri}

For an institutional subscription to digitalized issues, please contact Erudit.

Visit our website for Notes to contributors or to subscribe:

\section{www.riir.ulaval.ca}

\section{RELATIONS INDUSTRIELLES INDUSTRIAL RELATIONS}

Pavillon J.-A.-DeSève

1025, avenue des Sciences-Humaines Bureau 3129, Université Laval Québec (Québec) Canada G1V 0A6 TÉLÉPHONE : (418) 656-2468 COURRIEL / E-MAIL : relat.ind@rlt.ulaval.ca

\section{www.riir.ulaval.ca}

